\title{
NUCLEAR ANTIGENS FOLLOW DIFFERENT PATHWAYS INTO DAUGHTER NUCLEI DURING MITOSIS IN EARLY DROSOPHILA EMBRYOS
}

\author{
MANFRED FRASCH, DAVID M. GLOVER• \\ AND HARALD SAUMWEBER \\ Max-Planck-Institut für Entwicklungsbiologie, Spemannstrasse 35, 74 Tübingen, \\ Federal Republic of Germany
}

\begin{abstract}
SUMMARY
In the early embryonic development of Drosophila melanogaster, there is a series of 13 rapid and highly synchronous nuclear divisions. We have used a collection of monoclonal antibodies to follow the re-distribution of nuclear antigens into daughter nuclei at this developmental stage by indirect immunofluorescence microscopy. The antigens fall into several categories in terms of the pathways that are followed at mitosis. At one extreme is a group of antigens that remains continuously associated with the DNA throughout all the mitotic phases. At the other extreme, another group of antigens is excluded from the nucleus at prophase, and does not associate with the nucleus again until late telophase. One antigen, which becomes incorporated into the nucleolus at cellularization after the thirteenth division, becomes associated with the chromosomes during mitosis, but not until anaphase. Several different antibodies stain a diamond-shaped compartment that develops over the spindle at anaphase. The distribution of antigens within this spindle compartment shows some variation: one antigen appears to be present at higher concentrations in the central region of the spindle; others appear in three quite distinct areas corresponding to the positions of the new daughter nuclei and the old parental nucleus. Yet another antibody gives uniform staining of the spindle compartment. This antibody also recognizes a protein present in centrosomes.
\end{abstract}

\section{INTRODUCTION}

The embryo of Drosophila melanogaster is a syncytium for about the first $2 \mathrm{~h}$ of development, during which time there are 13 nuclear divisions. The first nine divisions occur within the interior of the embryo, with a cycle time of about $10 \mathrm{~min}$ (Zalokar \& Erk, 1976). Most of the nuclei then migrate towards the cortex of the embryo. The last four divisions take place just beneath the surface and have cycle times of 9, 10, 12 and $21 \mathrm{~min}$ (Foe \& Alberts, 1983). The exceptions are the yolk nuclei, which remain in the central part of the embryo and do not divide after the tenth nuclear cycle, but instead become polyploid (Foe \& Alberts, 1983). Mitosis is not perfectly synchronous, but rather waves of mitoses originate from the poles of the embryo speading towards the middle. Consequently, it is possible to observe several of the mitotic phases in a single embryo. Fluorescent probes have been used to follow

- Permanent address: Cancer Research Campaign, Eukaryotic Molecular Genetics Research Group, Department of Biochemistry, Imperial College of Science and Technology, London SW7 2AZ, England.

Key words: Drosophila embryos, mitosis, nuclear antigens. 
the behaviour of actin, myosin and a component of the nuclear envelope, lamin (Warn et al. 1980; Fuchs et al. 1983; Karr \& Alberts, unpublished). A recent study using monoclonal antibodies that recognize different groups of nuclear envelope antigens indicates that when lamin is dispersed into the cytoplasm during prometaphase, two other groups of nuclear envelope antigens are maintained within the nuclear compartment. These three groups of antigens subsequently follow different dynamics in the re-assembly of the nuclear envelope around the daughter nuclei (Frasch \& Saumweber, 1985). A recent electron-microscopic study has shown that nuclear pore complexes are lost from the nuclear envelope during pro-metaphase. The membrane subsequently develops large fenestrae and elongates to envelop the spindle throughout the mitotic process (Stafstrom \& Staehelin, 1985). We were interested to learn how other nuclear antigens are re-distributed into daughter nuclei in relation to any compartments that might be bounded by these membranes. We describe experiments in which we have used a collection of monoclonal antibodies against nuclear proteins (Saumweber et al. 1980; Frasch, 1985) to follow the timedependent distribution of various nuclear antigens during mitosis.

\section{MATERIALS AND METHODS}

\section{Antibodies}

Two libraries of monoclonal antibodies were used in this study: the first was established using nuclear fractions of Drosophila Kc cells (Saumweber et al. 1980); the second using nuclear fractions of 1- to 15-h-old embryos (Frasch, 1985).

TRITC-conjugated goat anti-mouse immunoglobulin $\mathrm{G}(\mathrm{IgG})(\mathrm{H}+\mathrm{L})$ was purchased from Capel, Cochranville, USA. Affinity-purified, peroxidase-conjugated goat anti-mouse $\operatorname{lgG}(\mathrm{H}+\mathrm{L})$ was obtained from Jackson Immunoresearch Inc., Avondale, PA, USA.

\section{Indirect immunofluorescence}

The P2 strain of Drosophila melanogaster (Allis et al. 1977) was used throughout this study. Whole mounts and sections of embryos were prepared for immunofluorescent staining as described by Dequin et al. (1984). Salivary glands were squashed and fixed for immunofluorescent staining as described by Saumweber et al. (1980), except that the phosphate buffer was substituted by buffer A (15 mM-Tris $\cdot \mathrm{HCl}, \mathrm{pH} 7 \cdot 4,60 \mathrm{~mm}-\mathrm{KCl}, 15 \mathrm{~mm}-\mathrm{NaCl}, 1.5 \mathrm{~mm}$-spermine, $0.5 \mathrm{~mm}$ spermidine). Buffer B (10 mM-potassium phosphate, $\mathrm{pH} 6 \cdot 8 ; 45 \mathrm{mM}-\mathrm{KCl}, 2 \mathrm{~mm}-\mathrm{MgCl}_{2}$ ) was used to stain salivary glands with antibodies Bm10 and By187.

\section{RESULTS}

\section{Antigens continuously associated with the chromosomes}

The availability of libraries of monoclonal antibodies, produced in this laboratory against nuclear proteins of Drosophila Kc cells and early embryos (Saumweber $e t$ al. 1980; Frasch, unpublished data), has enabled us to follow the partitioning of nuclear antigens into daughter nuclei during mitosis in early embryogenesis. The antibody At17 is one of several that remain continuously associated with DNA during the mitotic process. The staining of a field of prophase nuclei in a whole-mount preparation of Drosophila embryos is shown in Fig. 1. The localization of the antibody indicated by indirect immunofluorescence coincides with the fluorescence 
At 17

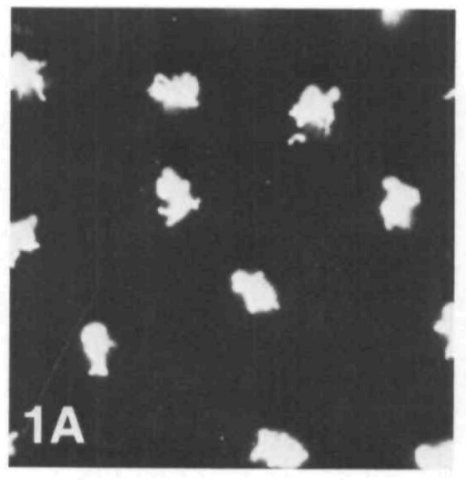

DNA

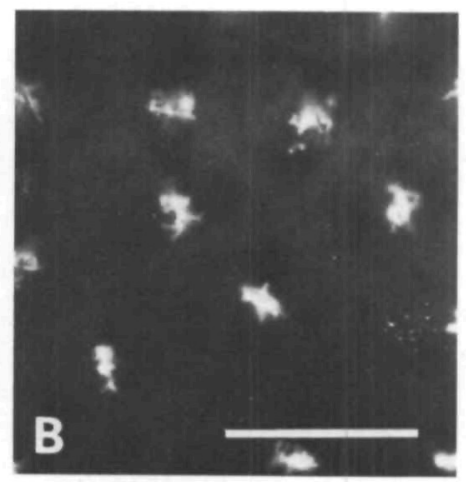

Fig. 1. The staining of pro-metaphase chromosomes in a whole-mount preparation of a blastoderm embryo with the antibody At17. Antibody staining is shown in A and DNA staining by Hoechst 33258 is shown in B. Bar, $30 \mu \mathrm{m}$.

of the DNA-Hoechst complex in all the mitotic phases. Thus, as shown in Fig. 1, the At17 antigen is associated with the condensing chromosomes at prophase. The antigen remains associated with the condensed chromosomes throughout anaphase and is subsequently uniformly distributed over the de-condensing chromosomes at telophase. We have also observed this pattern with antibodies Bj15 and By 187 against histones $\mathrm{H} 1$ and $\mathrm{H} 2 \mathrm{a}$, respectively; and with two other monoclonal antibodies, P12 and P2, against non-histone proteins. This latter group recognizes different proteins as judged by the results of Western blotting and also by the distribution of the antigens on polytene chromosomes (M. Frasch \& H. Saumweber, unpublished data; see also Table 1).

A similar but not identical pattern is given by our monoclonal antibody Bm10 that recognizes the A+T-rich binding protein D1 (Alfageme et al. 1976). Our observations using $\mathrm{Bm} 10$ indicate that this antigen is associated with the DNA throughout mitosis, but is enriched in the centromeric regions of chromosomes as would be expected from the known properties of the D1 protein (Fig. 2).

\section{Nuclear antigens that become cytoplasmic during mitosis}

The behaviour of this group of antigens is in contrast to that of the group described above. They are antigens that during interphase are contained within the nucleus, but with condensation of the chromosomes in prophase and metaphase they are redistributed into the cytoplasm. The association with cytoplasm persists throughout anaphase, and only in late telophase does the antigen begin to reassociate with the nucleus. One such example is shown in Fig. 3, for the case of the antigen recognized by antibody L62. By the time the metaphase plate has formed, the pattern of staining by the antibody has changed from being almost exclusively nuclear to one in which the antibody is excluded from the nucleus, with the exception of a faint residual staining over both sides of the metaphase plate (see the 'metaphase' panels in Fig. 3). 


\section{Bm 10}
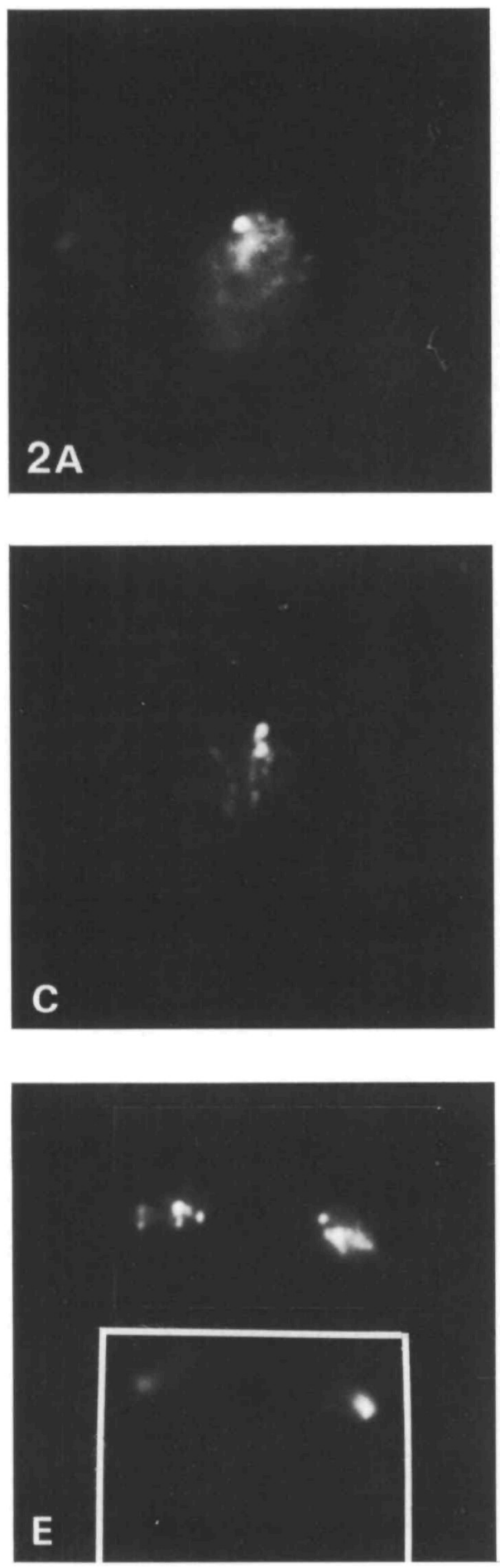

DNA
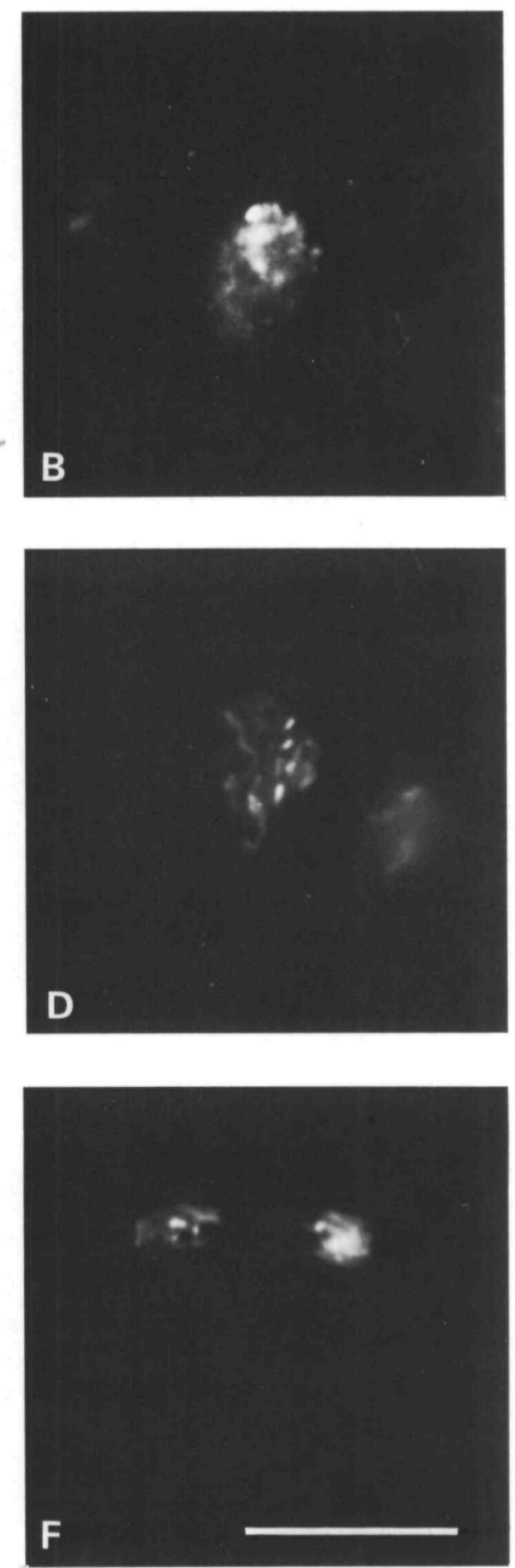


\section{L62}
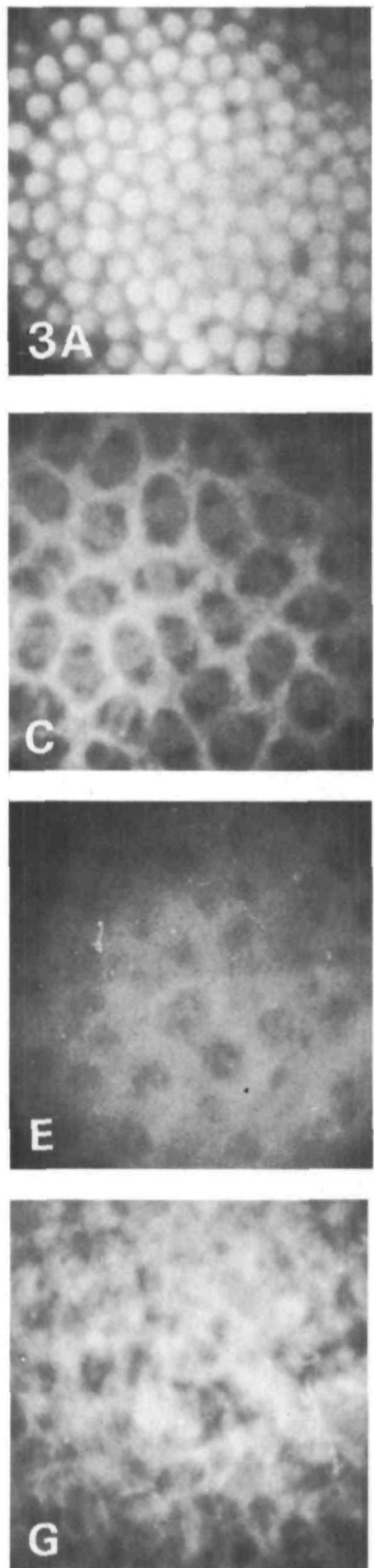

DNA
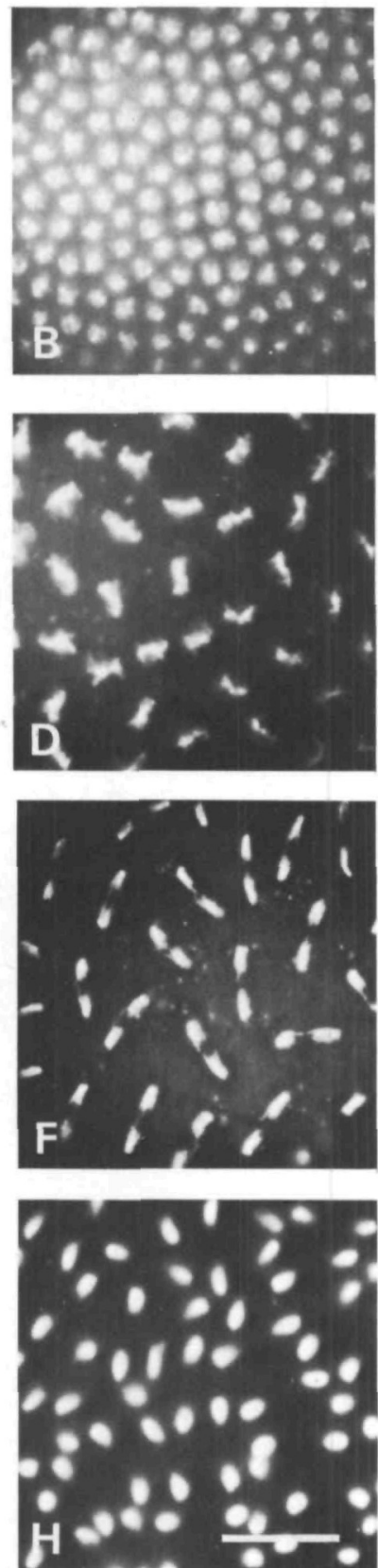

Fig. 3. The staining of whole-mount preparations of blastoderm embryos by antibody L62. Antibody staining is shown in the left panels, DNA staining in the right panels. Bar, $30 \mu \mathrm{m}$. A,B, interphase; C,D, metaphase; E, F, anaphase; $G, H$, telophase.

Fig. 2. The staining of mitotic chromosomes by antibody Bm10 in sectioned preparations of blastoderm embryos. The left panels show antibody staining, and the right panels, DNA staining. The inset in E shows antibody staining of the same field focused on the centromeric regions of the chromosomes. Bar, $15 \mu \mathrm{m}$. A,B, interphase; $C, D$, prometaphase; $E, F$, anaphase. 
The 'hole' formerly occupied by the parental interphase nucleus, and revealed by the antibody staining, elongates around the spindle during anaphase (Fig. 3). At telophase it is difficult to see the distinction between the nuclear and cytoplasmic compartments by antibody staining.

We have observed similar immunofluorescent staining given by seven other monoclonal antibodies (At5, Bs15, Bj43, V20, P4, T37, Y55) that recognize unrelated nuclear antigens. The antigens recognized by this set of antibodies are quite diverse in their properties and differ in their molecular weights as judged by Western blotting (Table 1). They include proteins that associate with interbands of polytene chromosomes (recognized by antibody Y55), proteins that are associated with bands of polytene chromosomes (recognized by antibody Bj43), proteins associated with puffs on polytene chromosomes (recognized by antibody Bs15), and proteins associated with a small number of distinct polytene chromosome regions (recognized by antibody At5). The antibody $\mathrm{Bj} 43$, shows a contrasting pattern in the cleavage stages of embryogenesis, in which the Bj43 antigen is associated with chromosomes throughout mitosis. During the blastoderm stages, however, its staining pattern resembles that given by antibody L62 shown in Fig. 3, but even here there is a small residual amount of chromosomal staining.

\section{An antigen that migrates onto the chromosomes after metaphase}

Fig. 4 shows blastoderm nuclei in various stages of the mitotic cycle stained with the antibody $\mathrm{Aj} 1$. Aj1 recognizes a nuclear antigen that, as the chromosomes condense in prophase (Fig. 4A,B), continues to stain an area corresponding to the position of the former interphase nucleus. This contrasts with the behaviour of the antibodies described in the first section of the results, such as At17, which stains condensed chromosomes at these phases (Fig. 1). The staining of a broader area by antibody Ajl persists during the formation of the metaphase plate. The antigen appears to be more concentrated around the metaphase plate and the metaphase chromosomes themselves are not stained. We have previously observed similar staining of a nuclear 'compartment' during prophase and metaphase with antibodies $\mathrm{Bx} 34$ and Bv38, which recognize antigens of the interphase nuclear envelope (Frasch \& Saumweber, 1985). This is at a time when staining of the nuclear envelope by antilamin antibodies has become extremely weak (see also Discussion). The pattern of staining by the $\mathrm{Aj} 1$ antibody changes as the mitotic cycle progresses. As the chromosomes begin to segregate during anaphase the $\mathrm{Aj} 1$ antigen undergoes redistribution to associate with the chromosomes per se. In addition, there is some weak staining of the region between the daughter chromosomes at anaphase (see Fig. 4).

The $\mathrm{Aj} 1$ antigen is distributed throughout interphase nuclei until cellularization at stage 14. Following cellularization, the staining becomes restricted to the nucleolus. The nucleolar staining is much weaker in intensity than the nuclear staining seen during cleavage. Nucleolar staining persists throughout all subsequent stages of development and is also seen in polytene cells (data not shown). Even when localized in the nucleolus of diploid cells, the $\mathrm{Aj} 1$ antigen undergoes a similar re-distribution during mitosis. However, until this time, the staining of interphase nuclei and of 
anaphase chromosomes is equally intense, whereas subsequently the staining of mitotically dividing cells is stronger than that of the interphase nucleolus. Areas of mitosis such as occur within the invaginations formed during gastrulation,

Table 1. The molecular weights and polytene chromosome distribution of antigens recognized by the antibodies used in this study

\begin{tabular}{cccc}
\hline & $\begin{array}{c}\text { Molecular weight } \\
\text { of antigen } \\
\left(\times 10^{-3}\right)\end{array}$ & $\begin{array}{c}\text { Distribution on } \\
\text { polytene chromosomes }\end{array}$ & Reference \\
\hline
\end{tabular}

Antigens continuously associated with chromatin

\begin{tabular}{|c|c|c|c|}
\hline At 17 & $\begin{array}{l}\text { Multiple bands, } \\
\text { major band at } 40\end{array}$ & Bands & This work \\
\hline P12 & 29 & Bands & Saumweber et al. (1980) \\
\hline By187 & $\underline{15}, 25$ & Bands & Frasch (1985) \\
\hline Bj15 & $\overline{32}$ & Bands & Frasch (1985) \\
\hline $\mathrm{P2}$ & n.d. & n.d. & This work \\
\hline $\mathrm{Bm} 10$ & 60 & $\begin{array}{l}\text { Overall staining of } \\
\text { chromosomes with } \\
\text { some sites showing } \\
\text { variable intensity }\end{array}$ & $\begin{array}{l}\text { Frasch (1985); } \\
\quad \text { Alfageme } \text { et al. (1976) }\end{array}$ \\
\hline
\end{tabular}

Antigens that become cytoplasmic during mitosis

$\begin{array}{llll}\text { L62 } & \underline{121}, \underline{66}, \underline{40},+ \text { minor } & \text { Puffs and interbands } & \text { This work } \\ \text { bands } & 129,83, \underline{60}, \underline{58}, \underline{56 \cdot 5} & \text { Small number of bands } & \text { This work } \\ \text { At5 } & 80 & \text { Puffs and nucleolus } & \text { Frasch }(1985) \\ \text { Bs15 } & 68 & \text { Bands } & \text { Frasch }(1985) \\ \text { Bj43 } & 38 & \text { Puffs } & \text { Saumweber } \text { et al. }(1980) \\ \text { V20 } & 28 & \text { n.d. } & \text { This work } \\ \text { P4 } & 83 & \text { Puffs } & \text { Saumweber } \text { et al. }(1980) \\ \text { T37 } & 131 & \text { Interbands } & \text { This work }\end{array}$

Antigen that migrates onto the chromosomes after metaphase
Aj1
47
Nucleolus
Saumweber et al. (1980)

Antigens that remain in the spindle region

\begin{tabular}{|c|c|c|c|}
\hline $\mathrm{Bj} 6$ & 82000 & Puffs & Frasch (1985) \\
\hline W12 & n.d. & Puffs & This work \\
\hline W2 & 79,51 & Puffs & This work \\
\hline Bs11 & $\overline{150}, 94,80,73,40$ & $\begin{array}{l}\text { Dots or fibres along the } \\
\text { chromosomes }\end{array}$ & Frasch (198 \\
\hline
\end{tabular}

A nuclear antigen also associated with the centrasome

Bx63 185, 69, 58 Interbands Frasch (1985)

Molecular weights were determined by Western blotting onto total proteins from Kc cell nuclei fractionated by electrophoresis on 9-17\% SDS/polyacrylamide gels as described by Dequin $e t$ al. (1984). In cases where there are several bands, the major bands are underlined. The second antibody (goat anti-mouse) was used at a dilution of $1: 2000$. The analysis of the antigen distribution on polytene chromosomes was carried out as described by Saumweber $e t$ al. (1980). The TRITC-conjugated second antibody was used at a dilution of $1: 200$. 
Aj 1
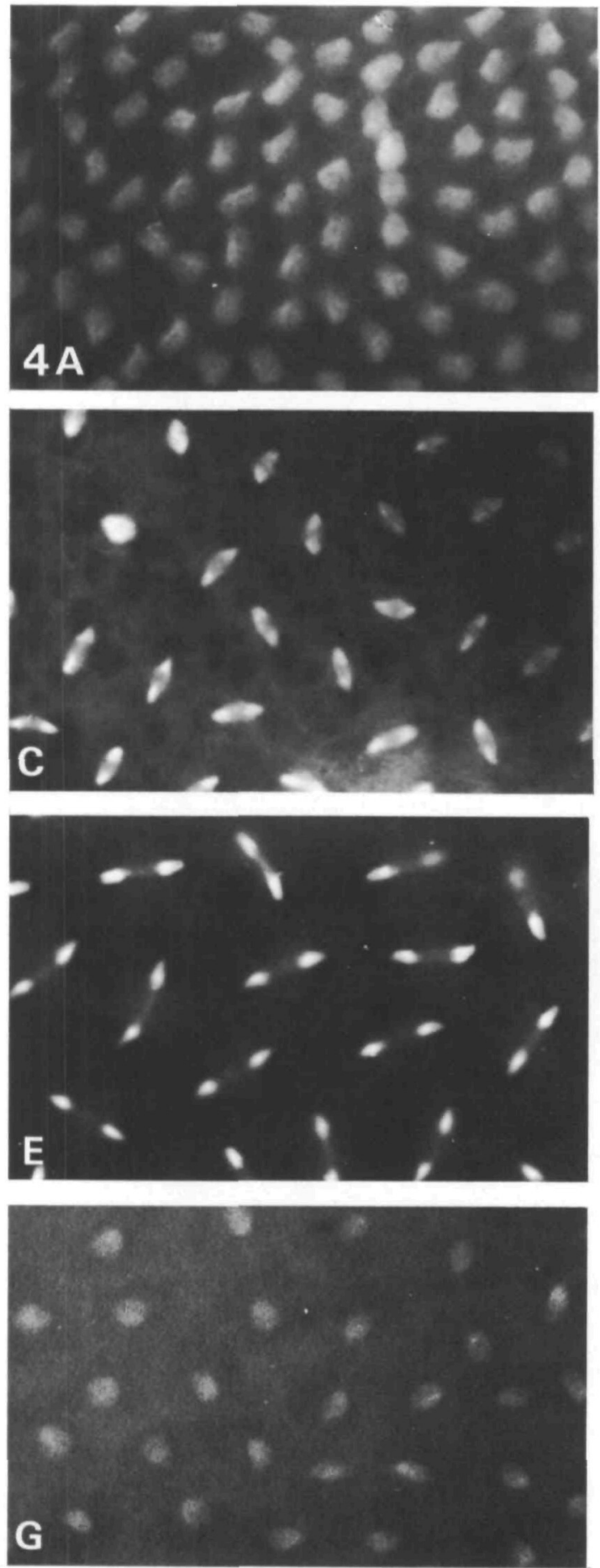

DNA
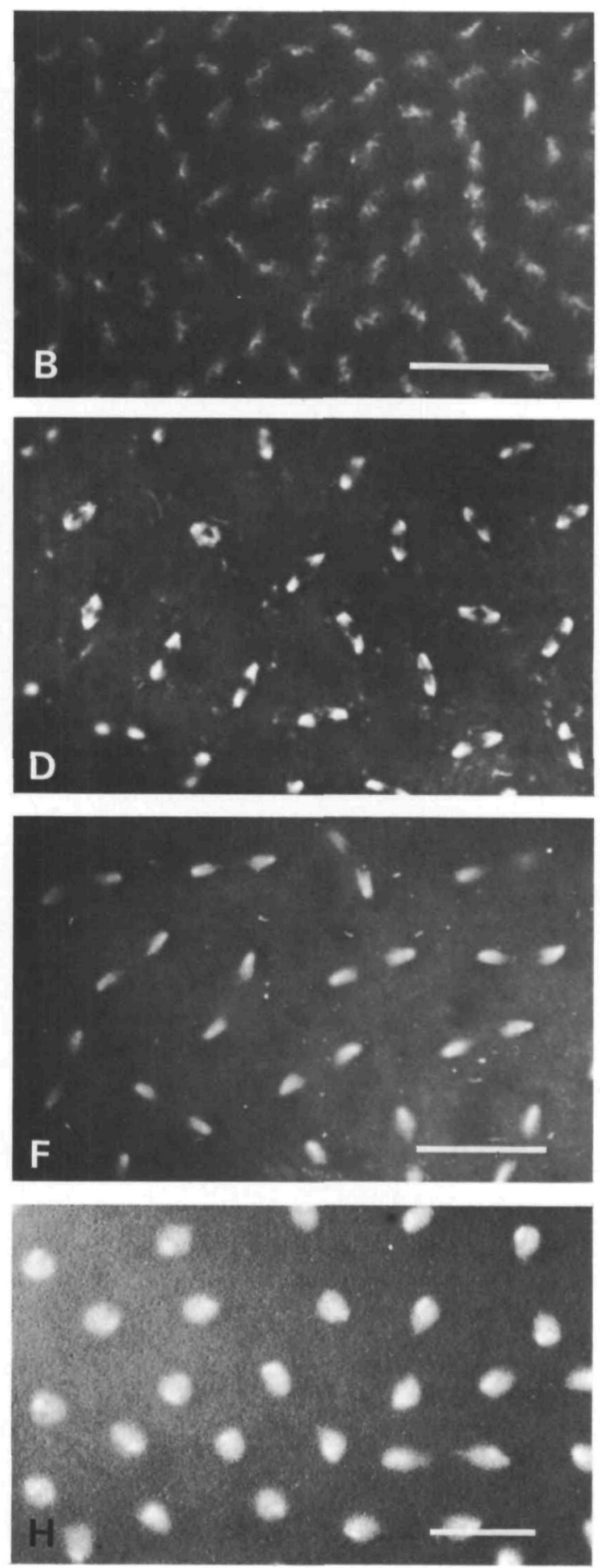
for example, can therefore easily be recognized by immunofluorescent antibody staining.

\section{Antigens that remain within the confines of the spindle region}

Another group of antibodies also stains the interphase nucleus and continues to stain the region formerly occupied by the nucleus as the chromosomes condense. They differ from the Aj1 antibody described above in that as the daughter chromosomes separate, the region stained by the antibodies elongates in the shape of the spindle structure. The antibody stains the area occupied by the spindle in early anaphase, but during late anaphase and early telophase, a tripartite structure develops in which there is staining over each set of daughter chromosomes and staining in the central region. This pattern is exemplified by antibodies $B j 6, W 12$ and W2. Examples of the staining given by antibodies $B \mathrm{j} 6$ and $\mathrm{W} 12$ are shown in Fig. 5, which shows whole-mount preparations in which there are mitotic gradients containing anaphase and telophase figures. Some telophase nuclei showing the tripartite staining pattern are indicated by arrowheads for the antibodies $\mathrm{Bj} 6$ and W12. In these same fields it is possible to recognize anaphase figures in which the antibody stains a diamond-shaped block corresponding to the spindle region. The staining of the different mitotic phases by the antibody $\mathrm{Bj} 6$ is shown in sectioned preparations in Fig. 6. This shows the diamond-shaped structure stained by the antibody in early anaphase, and how it develops into the tripartite structure in late anaphase. The three antibodies, $B j 6, W 12$ and $W 2$, recognize different antigens by the criteria of Western blotting and yet each stains puffs on polytene chromosomes (see Table 1).

The Bs 11 antigen shows a variation of this theme during anaphase, in which there is strong staining over the central part of the spindle region, with no concomitant staining over the chromosomal areas. This is shown in the whole-mount preparation in Fig. 5 and has also been observed in sectioned preparations (data not shown).

\section{A nuclear antigen also associated with the centrosome}

The Bx63 antibody stains both the nucleus and its associated centrosome. Staining of whole-mount preparations and of sectioned preparations of embryos at blastoderm stage with the Bx63 antibody is shown in Figs 7 and 8, respectively. The distribution of the 'nuclear component' during mitosis is reminiscent of that given by the previous group of antibodies, and persists in the region immediately surrounding the metaphase plates. It remains in this area during anaphase and appears confined within the region of the spindle. Subsequently, it is re-distributed between the telophase nuclei but without the formation of the tripartite pattern observed with antibody Bj6, for example (see above). The staining intensity of this 'nuclear component' becomes progressively weaker from metaphase onwards and at telophase the remaining Bx63 antigen is detected within the region formerly occupied by the spindle. The staining

Fig. 4. The staining of whole-mount preparations of blastoderm embryos by antibody Aj1. Antibody staining is shown in the left panels, DNA staining in the right panels. Bars, $A-F, 30 \mu \mathrm{m} ; \mathrm{G}, \mathrm{H}, 15 \mu \mathrm{m}$. A,B, pro-metaphase; $C-F$, anaphase; $\mathrm{G}, \mathrm{H}$, telophase. 
covers a larger area than the Hoechst-stained chromosomes, still linking the two daughter nuclei, but being constricted in its central part (see the cartoon on the right of E,F, Fig. 7).

Immunofluorescence
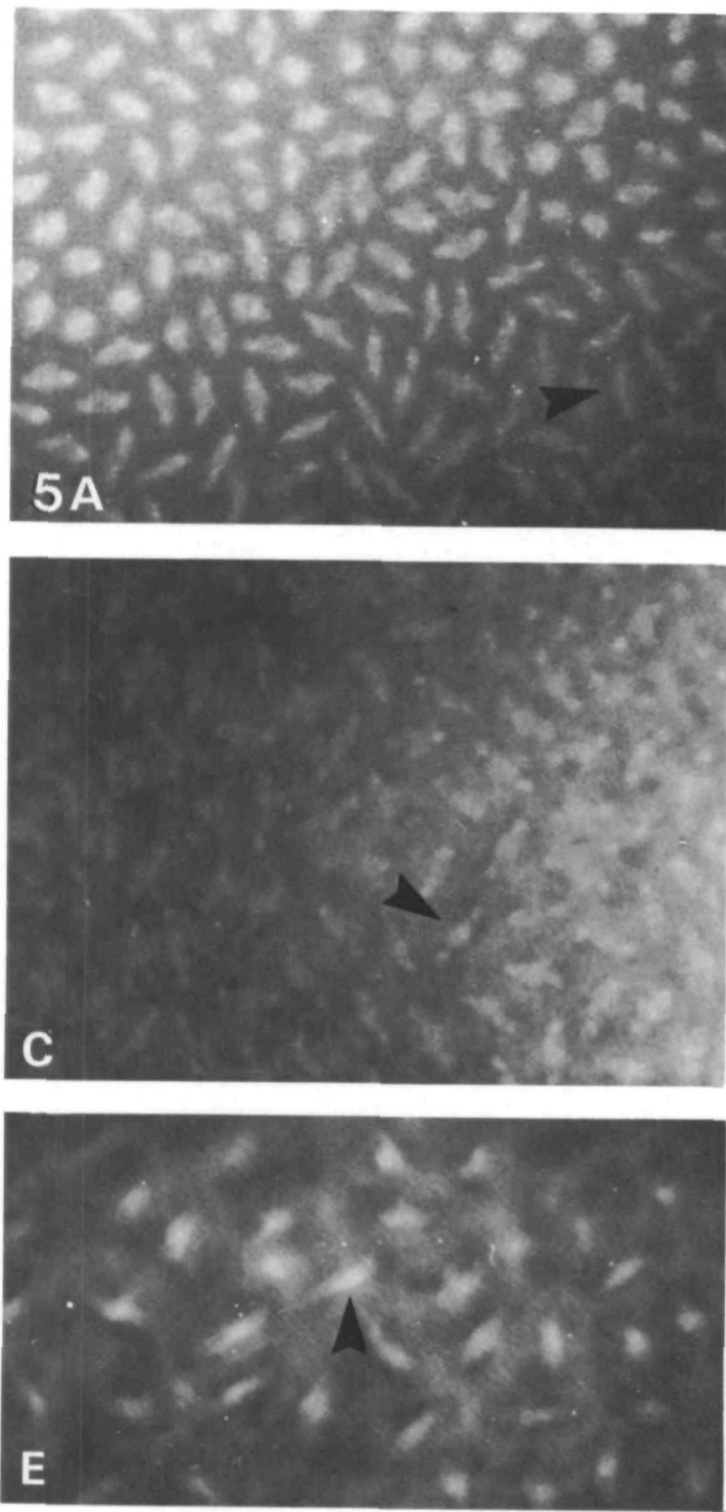

DNA-Hoechst fluorescence
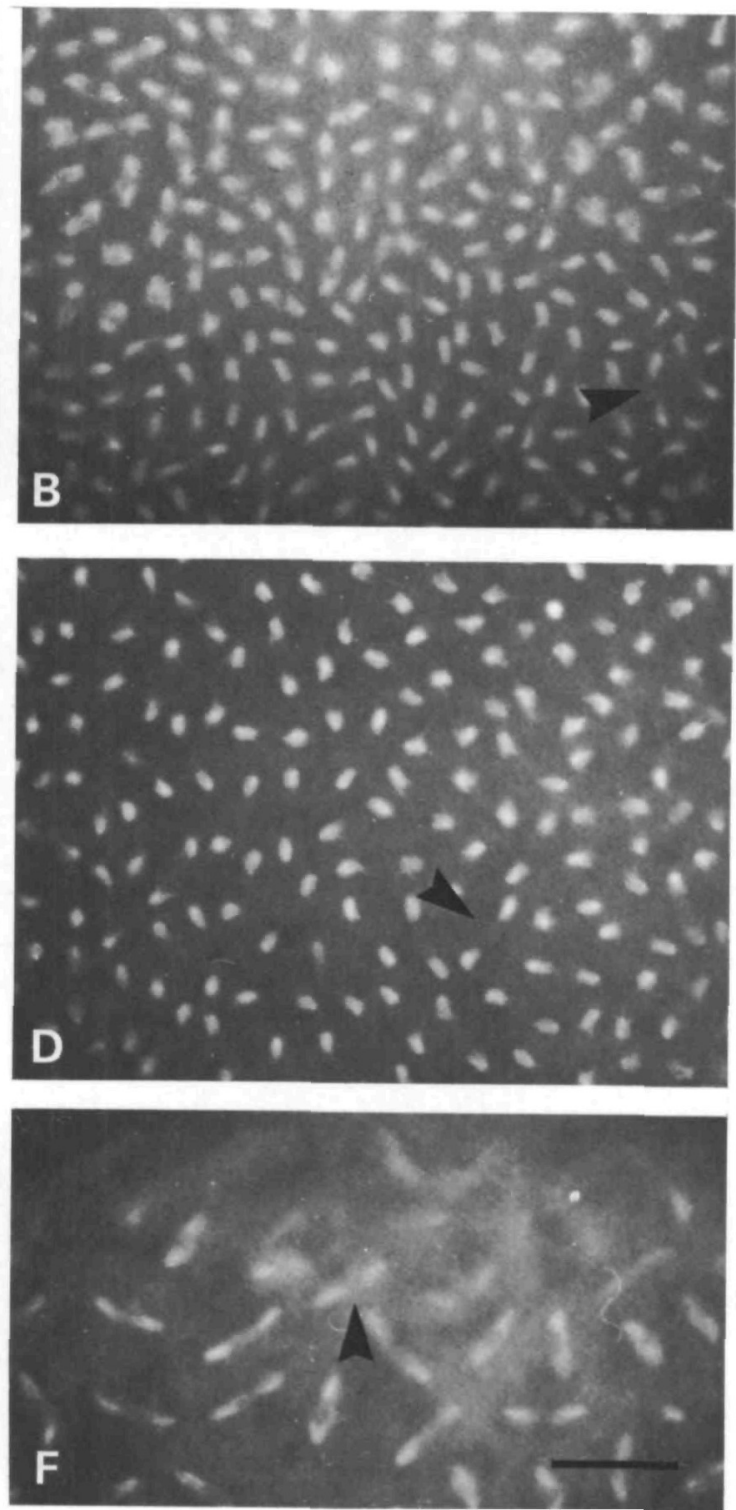

Fig. 5. The staining of mitotically dividing nuclei in whole-mount preparations of blastoderm embryos stained with the antibodies $\mathrm{Bj} 6, \mathrm{~W} 12$ and Bs11. A,B (Bj6 staining) shows a field of nuclei with a mitotic gradient from early to mid-anaphase; C,D (W12 staining), mid-anaphase to early telophase; and E,F, (Bs11 staining), mid- to lateanaphase. Antibody staining is shown in $A, C, E$ and 'DNA staining in $B, D, F$. The arrowheads point to corresponding anaphase figures in the antibody-stained and DNAstained fields. Bar, $30 \mu \mathrm{m}$. 
Centrosomal staining persists throughout all the mitotic phases. In the mitotic gradient between prophase and metaphase shown in Fig. 7A,B, the daughter centrosomes can be seen to migrate to either side of the metaphase plate (see the cartoon showing the superimposition of the chromosomes and centrosomes to the right of $A, B)$. The central panels of this figure show an anaphase field. In several areas of this field it can be seen that there is a tendency of centrosomes from adjacent spindles to pair in close juxtaposition. We observed in sectioned preparations that the
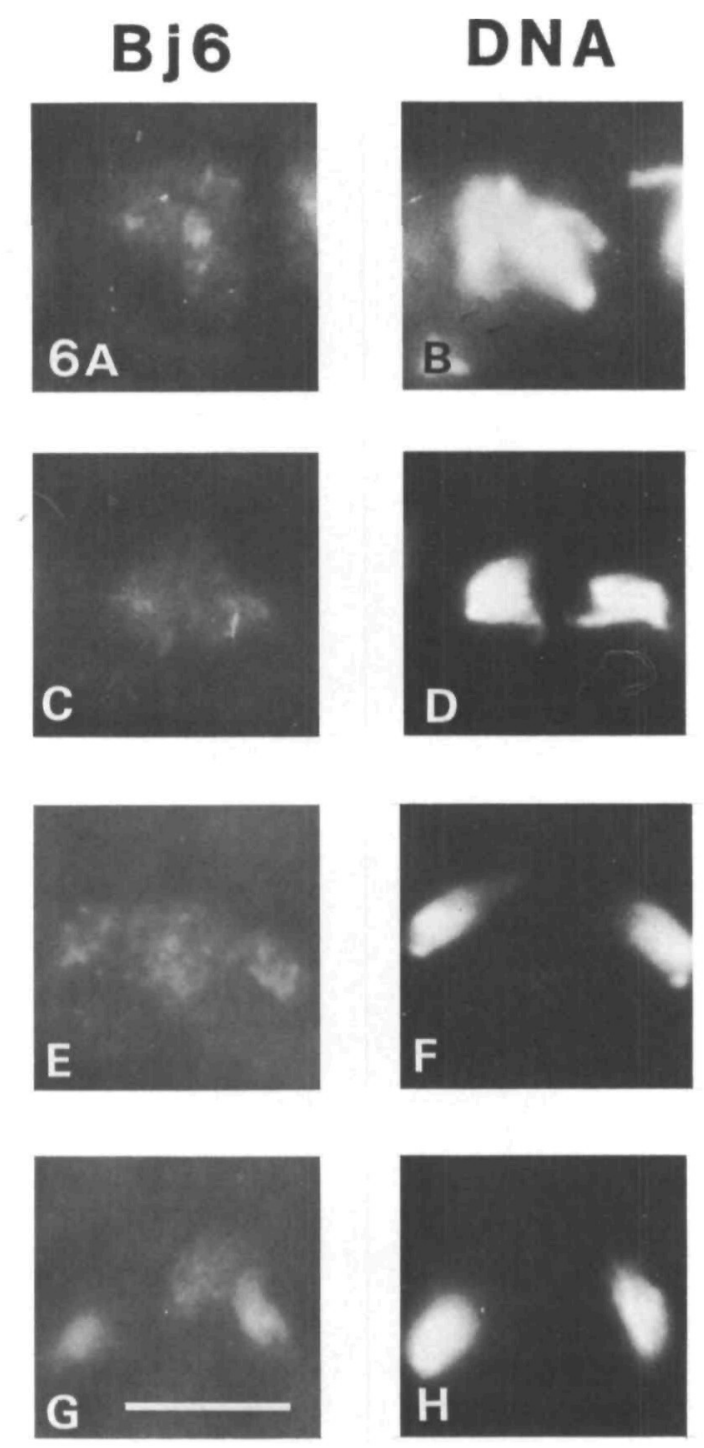

Fig. 6. The staining of mitotically dividing nuclei in the cortex of sectioned blastoderm embryos by antibody Bj6. Antibody staining is shown in A,C,E,G and DNA staining in $B, D, F, H$. Bar, $10 \mu \mathrm{m}$. A, B, metaphase; C,D, early anaphase; E,F, late anaphase; G,H, early telophase. 


\section{B $\times 63$}

\section{$7 \mathrm{~A}$}

C

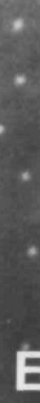

E
DNA

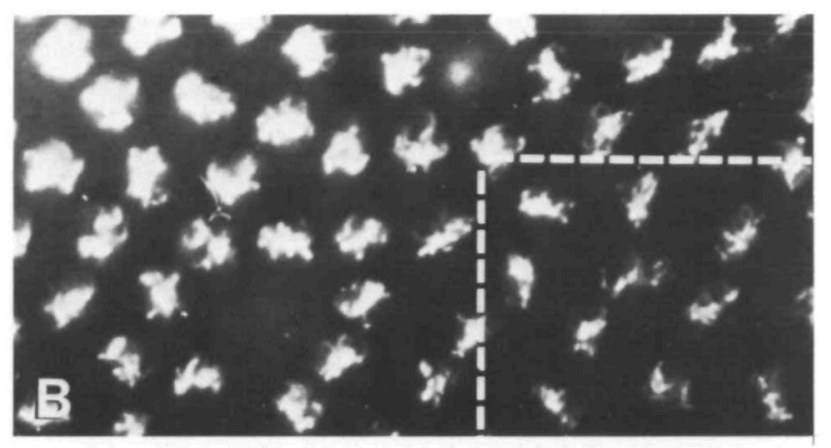

$\because \cdot \dot{H}^{*}$

. is

-

.4. . . .

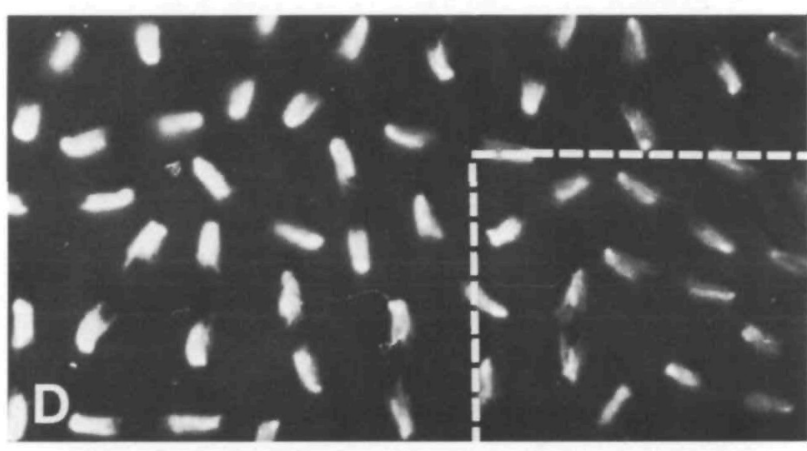

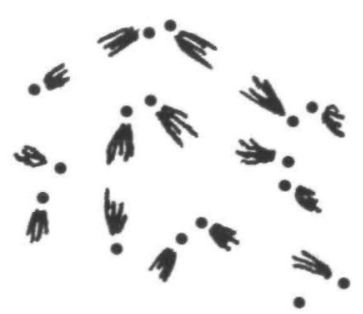

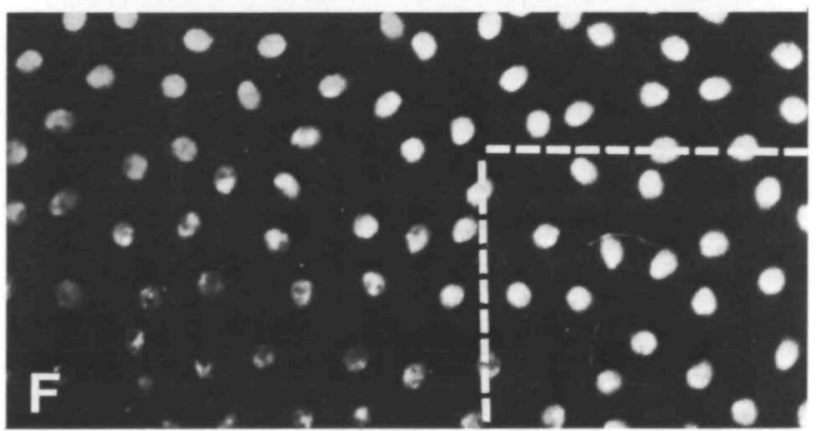

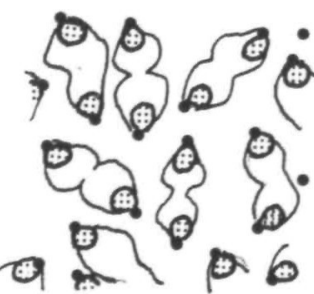




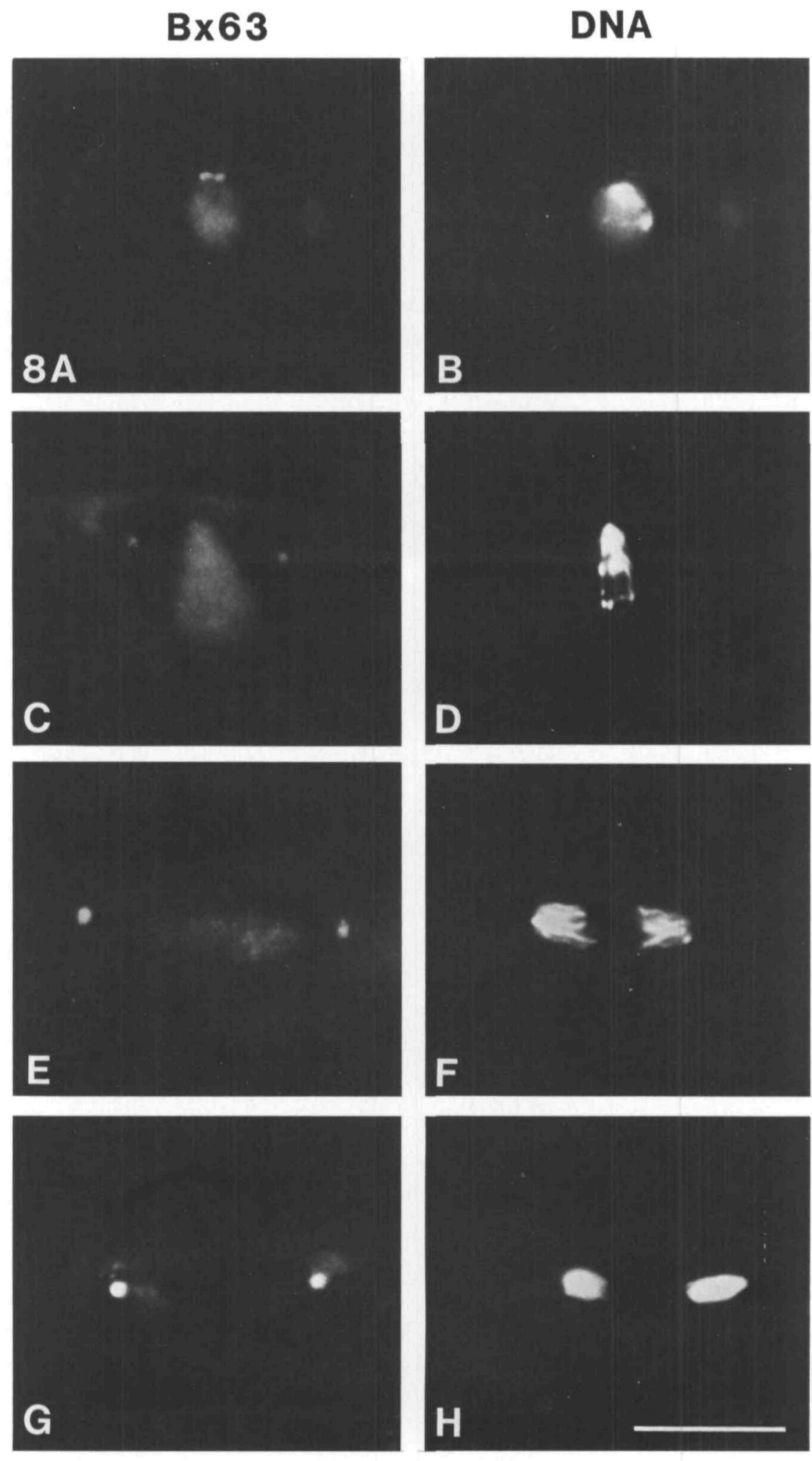

Fig. 8. For legend see p. 168 
centrosome is located between the plasma membrane and the interphase nucleus, adjacent to the centromeric region of the chromosomes. The centrosomes remain in the vicinity of the plasma membrane throughout the mitotic cycle. In cross-sections of embryos at late blastoderm it is as though two daughter nuclei were being pulled towards the plasma membrane.

The staining of the nucleus by antibody Bx63 varies during development. During oogenesis, the oocyte nucleus becomes more strongly stained than the nurse cell or follicle cell nuclei. The oocyte nucleus begins to be strongly stained at stage 3 of oogenesis and remains strongly stained until at least stage 10 of oocyte development (King et al. 1956). (At later stages we cannot observe the staining pattern since the antibody does not penetrate the vitelline membrane.) In cleavage embryos, the nucleus is not stained, but there is staining of the centrosomes and of the cytoplasm. Nuclear staining returns at the beginning of blastoderm at cell cycle 10 . At cell cycle 12 , the yolk nuclei in the interior of the embryos cease dividing and begin to become polyploid. At this stage we are no longer able to detect centrosomal staining adjacent to the yolk nuclei. We have examined the staining pattern given by the antibody in the polytene chromosomes of the salivary gland and find staining of a high proportion of interbands and a number of dots over the chromocentre (data not shown).

\section{DISCUSSION}

We are interested in knowing how nuclear antigens are partitioned between daughter nuclei at mitosis. The early embryonic stages of Drosophila development provide material highly suited to address this problem. The nuclear division cycles $10,11,12$ and 13 last for $9,10,12$ and $21 \mathrm{~min}$, respectively, and mitoses occur in parasynchronous waves, which can be readily observed in whole-mount or sectioned preparations of embryos (Foe \& Alberts, 1983). We have therefore used indirect immunofluorescence labelling to follow the behaviour of a number of nuclear antigens recognized by a collection of monoclonal antibodies isolated in this laboratory (Saumweber et al. 1980; Frasch, 1985). The functions of the majority of these antigens are unknown, but that they are distinct is apparent: from analyses by Western blotting; from the cytology of their staining of salivary gland chromosomes; and in some cases from additional biochemical fractionation procedures. We have observed that nuclear antigens follow a variety of pathways into daughter nuclei at mitosis. At one extreme there are antigens that are associated with DNA throughout

Fig. 7. The staining of whole-mount preparations of blastoderm embryos by antibody Bx63. Antibody staining is shown in $A, C, E$ and DNA staining in $B, D, F$. The lower right corner of each figure is represented in the cartoon drawings on the right, which show the superimposition of centrosome staining (depicted by dots) and DNA staining (depicted by heavy lines in A-D and by stippled areas in $E, F)$. In the cartoon for $E, F$, the boundary of the staining of the nuclear component recognized by Bx63 is shown by a thin line. $\times 700$. A, B, pro-metaphase; C,D, anaphase; E,F, telophase.

Fig. 8. The staining of mitotically dividing nuclei in the cortex of sectioned blastoderm embryos by antibody Bx63. Antibody staining is shown in A,C,E, G and DNA staining in B,D,F,H. Bar, $15 \mu \mathrm{m}$. A,B, early prophase; C,D, pro-metaphase; E, F, anaphase; G,H, early telophase. 
the mitotic cycle (see Fig. 1), at the other there are nuclear antigens that become cytoplasmic as the chromosomes condense in prophase (see Fig. 2). In between these extremes there are a number of possibilities. One antigen remains in the area occupied by the former nucleus up until metaphase, and then migrates onto the anaphase chromosomes. Another group of antibodies shows more complex patterns and stains a cellular compartment occupied by the spindle at anaphase. Finally, we describe an antigenic determinant present in both the nucleus and the centrosome, the nuclear component of which also stains the spindle compartment during anaphase.

Our observations point towards the existence of a compartment during prophase and metaphase that persists in the space formerly occupied by the interphase nucleus. Antigens recognized by several antibodies occupy this compartment, examples of which can be seen with Aj1 in Figs 3 and 4, with Bj6 in Fig. 6 and with antibody Bx63 in Figs 7 and 8 . We have also observed comparable staining patterns at these mitotic phases with antibodies Bx34 and Bv38 (Frasch \& Saumweber, 1985). The latter two monoclonal antibodies recognize determinants on high molecular weight components of the interphase nuclear envelope and stain the circumference of the nucleus brightly. This pattern changes markedly with chromosome condensation to give one in which the entire nuclear compartment is stained. This is in contrast to the behaviour of antibodies directed against the nuclear lamins (Fuchs et al. 1983), which also give strong staining of the nuclear envelope at interphase. At metaphase, the staining by the anti-lamin antibodies is very greatly reduced and the antigen is dispersed and excluded from the area formerly occupied by the interphase nucleus. Together these observations suggest that the nuclear envelope undergoes considerable structural change during prophase and metaphase. However, the nuclear compartment still appears to be separated from cytoplasm. Supporting evidence comes from direct observations in the electron microscope by Stafstrom \& Staehelin (1985). They have observed the persistence of the nuclear envelope throughout metaphase but with the loss of the nuclear pore complexes and the appearance of irregular fenestrae. The boundaries of a nuclear compartment can therefore be recognized well into metaphase. It seems that some nuclear antigens remain within this compartment where they may be tightly associated with chromosomes (e.g. the At17 antigen) or not (e.g. the antigen Bv38). Other antigens, for example L62, are specifically excluded from this compartment at mitosis.

The work of Stafstrom \& Staehelin (1985) indicates that the envelope around the metaphase nucleus persists throughout the mitotic process. It is seen to elongate and cover the entire spindle structure, but does not extend to the poles. At telophase, the new nuclear envelope begins to form around the de-condensing chromatin and is seen in the electron microscope as a structure that probably corresponds to the cuplike structures that are seen to form around the telophase nuclei when staining with an anti-lamin antibody (Fuchs et al. 1983) or Bx95 (Frasch \& Saumweber, 1985). This spindle envelope can persist until the new interphase nuclei are formed. The staining given by several of our antibodies is consistent with the idea that some of the antigens are retained within a spindle compartment, the boundary of which could be 
the spindle envelope. This is clearly seen for the staining given by antibodies $\mathrm{Bj} 6$, W12 and Bs11, each of which stains a diamond-shaped area over the spindle during early anaphase (see Fig. 6). The distribution of different antigens within this compartment shows some variation. At the late stages of anaphase, the $\mathrm{Bj} 6$ antigen shows separate association with a region corresponding to the site of the future daughter nuclei and with the central area of the spindle formerly occupied by the parental nucleus (see Fig. 6). A similar pattern is seen with antigens W2 and W12. The Bs11 antibody also stains the spindle compartment, but differs by showing a stronger staining of the central region with very little staining over the chromosomes. There is an alternative variation with the staining given by the Aj1 antibody, which, at anaphase, is intense over the chromosomes and weak in the central part of the spindle. Weak staining of the spindle compartment is also given by the antibody $\mathrm{Bx} 63$ in addition to strong staining of the centrosomes. This spindle-staining component of the $\mathrm{Bx} 63$ antigen probably corresponds to a $\mathrm{Bx} 63$ component seen within the parental interphase nucleus. The spindle staining is more uniform in contrast to the staining with other antibodies, and at telophase it divides into two regions associated with but occupying a larger area than the daughter nuclei. The distribution of nuclear antigens within the spindle compartment is therefore quite complex, reflecting the complex dynamic process of disassembly and reassembly of the nucleus at mitosis.

The Aj1 antigen falls into a separate category in that it changes its distribution in the nuclear and spindle compartments during mitosis. At metaphase in cleavage and blastoderm embryos, the Aj1 antibody stains the nuclear region surrounding the chromosomes. During anaphase, however, the Aj1 antigen appears to migrate onto the condensed chromosomes and the predominant antibody staining is seen over chromosomal regions. Following cellularization at stage 14 of embryogenesis, there is a significant increase in the transcription of the zygotic nuclei (McKnight \& Miller, 1976; Anderson \& Lengyel, 1979). Concomitant with this is the appearance for the first time of discrete nucleoli. At this developmental stage the antibody Aj1 begins to stain the nucleolus rather than the whole nucleus of interphase cells. This nucleolar staining is much weaker, suggesting that antigenic sites are being masked either by protein modification or by the assembly of the antigen into the nucleolus. Once the nucleus has disintegrated the immunofluorescent staining becomes brighter again in the following mitotic phases.

It could be that a number of nucleolar proteins follow this pathway during the disruption of the nucleolus that occurs at mitosis. Several proteins of the nucleolus can be stained using silver nitrate. The behaviour of these argyrophilic proteins was recently followed in mitotically dividing human breast cancer cells (Ploton et al. 1985). These workers observed that the argyrophilic proteins became localized around the periphery of the chromatin mass during anaphase. Using a monoclonal antibody, Hugle et al. (1985) have studied the mitotic behaviour of an acidic protein, 'ribocharin', associated with pre-ribosomal particles in Xenopus. In somatic cells undergoing mitosis, this protein transiently forms a coat on the surfaces of all chromosomes. It is subsequently reincorporated into nucleoli during late 
telophase. This pattern of protein re-distribution is not restricted to components of the nucleolus. McKeon et al. (1984) have described an antiserum from a patient with systemic lupus erythematosis that recognizes evolutionarily conserved determinants of the nuclear envelope of mammalian cells and Drosophila cells. During mitosis, these determinants associate with the periphery of the condensed chromosomes. This perhaps points to a more general pathway whereby proteins located in different regions of the interphase nucleus can 'hitch a ride' into daughter nuclei on the segregating chromosomes.

The staining of the centrosomes by the Bx63 antibody is striking. The antigen appears to be provided maternally, in that it accumulates within the oocyte nucleus. Furthermore, data from Western blots of total embryonic protein from different stages (not shown) indicate the antigen is present in sufficiently large amounts in the early embryo for embryonic development. Centrosomes are associated with all the dividing cells in the embryo, but are noticeably absent from the yolk cell nuclei that cease dividing and become polyploid. Our observations on sectioned embryos indicate that the centrosomes are located immediately beneath the plasma membrane, being directly above interphase nuclei. This is consistent with the predictions from detailed studies of the organization of the cytoskeleton in early Drosophila embryos using monoclonal antibodies against actin and tubulin (Karr \& Alberts, unpublished). The centrosomes divide during early prophase but at this stage and throughout mitosis they remain in closer association with the cortical region of the cytoplasm than does the chromatin. Western blots indicate three major antigenic polypeptides that have molecular weights of 185,69 and $58\left(\times 10^{3}\right)$. We do not yet know whether the polypeptides are distributed equally between nuclei and centrosomes. This problem together with other properties of the antigen is under further investigation.

We thank Dr Friedrich Bonhoeffer for his encouragement and for stimulating discussions. D.M.G. holds a Career Development Award from the Cancer Research Campaign and was supported by short-term fellowships from the European Molecular Biology Organisation for visits to Tübingen in 1984 and 1985.

\section{REFERENCES}

Alfageme, C. R., RudKIN, G. T. \& Cohen, L. H. (1976). Locations of chromosomal proteins on polytene chromosomes. Proc. natn. Acad. Sci. U.SA. 373, 2038-2042.

Allis, C. D., Waring, G. L. \& MaHowald, A. P. (1977). Mass isolation of pole cells from Drosophila melanogaster. Devl Biol. 56, 372-381.

ANDERSON, K. V. \& LENGYel, J. A. (1979). Rates of synthesis of major classes of RNA in Drosophila embryos. Devl Biol. 70, 217-231.

Dequin, R., Saumweber, H. \& Sedat, J. W. (1984). Proteins shifting from the cytoplasm into the nuclei during early embryogenesis of Drosophila melanogaster. Devl Biol. 104, 37-48.

FOE, V. E. \& AlberTS, B. M. (1983). Studies of nuclear and cytoplasmic behaviour during the five mitotic cycles that precede gastrulation in Drosophila embryogenesis. F. Cell Sci. 61, 31-70.

Frasch, M. (1985). Doctoral dissertation. Eberhard-Karls-Universität, Tübingen.

Frasch, M. \& SAUmWEBer, H. (1986). Developmental and mitotic behaviour of three different groups of nuclear envelope antigens of Drosophila melanogaster (in press).

Fuchs, J.-P., Giloh, H., Kuo, C.-H., Saumweber, H. \& Sedat, J. (1983). Nuclear structure: determination of the fate of the nuclear envelope in Drosophila during mitosis using monoclonal antibodies. F. Cell Sci. 64, 331-349. 
Hugle, B., Scheer, B. \& Franke, W. W. (1985). Ribocharin - a nuclear $M_{\mathrm{r}} 40,000$ protein specific to precursor particles of the large ribosomal subunit. Cell 41, 615-627.

King, R. C., Rubinson, A. L. \& SMITH, R. F. (1956). Oogenesis in adult Drosophila melanogaster. Growth 20, 121-157.

McKeon, F. D., Tuffanelli, D. L., Kobayashi, S. \& Kirschner, M. W. (1984). The redistribution of a conserved nuclear envelope protein during the cell cycle suggests a pathway for chromosome condensation. Cell 36, 83-92.

MCKNiGht, S. L. \& Miller, O. L. (1978). Ultrastructural patterns of RNA synthesis during early embryogenesis of Drosophila. Cell 8, 305-319.

Ploton, D., Menager, M. \& Adnet, J. J. (1985). Simultaneous ultrastructural localisation of Ag-NOR (Nucleolar Organizer Region) proteins and ribonucleoproteins during mitosis in human breast cancerous tissue. F. Cell Sci. 74, 239-256.

Saumweber, H., Symmons, P., Kabisch, R., Will, H. \& Bonhoeffer, F. (1980). Monoclonal antibodies against chromosomal proteins of Drosophila melanogaster. Chromosoma 80, 253-275.

Stafstrom, J. P. \& Staehelin, L. A. (1985). Dynamics of the nuclear envelope and of nuclear pore complexes during mitosis in the Drosophila embryo. Eur. J. Cell Biol. 34, 179-189.

WARN, R. M., Bullard, R. \& MaGRath, R. (1980). Changes in the distribution of cortical myosin during the cellularisation of the Drosophila embryo. J. Embryol. exp. Morph. 57, 167-176.

ZALOKAR, M. \& ERK, I. (1976). Division and migration of nuclei during early embryogenesis of Drosophila melanogaster. 7. Microsc. Biol. Cell. 25, 97-106.

(Received 2 October 1985 - Accepted 22 October 1985) 